\title{
Habilidades socioemocionales en las comunidades educativas: aportes para la formación integral de los y las docentes
}

Aristulle, Patricia del Carmen; Paoloni-Stente, Paola Verónica

Habilidades socioemocionales en las comunidades educativas: aportes para la formación integral de los y las docentes

Revista Educación, vol. 43, núm. 2, 2019

Universidad de Costa Rica, Costa Rica

Disponible en: http://www.redalyc.org/articulo.oa?id=44058158005

DOI: https://doi.org/10.15517/revedu.v43i2.28643

Esta obra está bajo una Licencia Creative Commons Atribución-NoComercial-SinDerivar 3.0 Internacional. 


\section{Habilidades socioemocionales en las comunidades educativas: aportes para la formación integral de los y las docentes}

\section{Socio-Emotional Skills in Educational Communities: Contributions to Integral Teacher Training}

Patricia del Carmen Aristulle

Universidad Nacional de Rio Cuarto- CONICET,

Argentina

patriciaaristulle@gmail.com

(iD) http://orcid.org/0000-0001-7871-9179

Paola Verónica Paoloni-Stente

Universidad Nacional de Río Cuarto- CONICET,

Argentina

paopaoloni17@hotmail.com

(D) http://orcid.org/0000-0002-9384-010X
DOI: https://doi.org/10.15517/revedu.v43i2.28643

Redalyc: http://www.redalyc.org/articulo.oa?id=44058158005
Recepción: 21 Abril 2017

Aprobación: 22 Abril 2019

\section{Resumen:}

Este trabajo se orienta a describir y analizar el rol de las emociones y de las habilidades socioemocionales en el proceso de conformación de una comunidad educativa. La propuesta se implementó en los Profesorados de Educación Inicial y de Educación Primaria del Instituto Superior de Formación Docente San José (Córdoba, Argentina). La escuela constituye un ámbito favorable para el desarrollo de aprendizajes que exceden los contenidos curricularmente prescriptos. Entre estos contenidos, se destaca el desarrollo de habilidades socioemocionales, un tópico que impacta favorablemente en la conformación de una comunidad educativa. Nace la pregunta, entonces, cómo se prepara el futuro profesorado para considerar en sus prácticas estas perspectivas amplias del enseñar y del aprender, que superen ampliamente las habilidades cognitivas y coloquen entre otras prioridades las habilidades socioemocionales, importantes para su quehacer profesional. El trabajo que se presenta, pretende promover una reflexión sobre la complejidad de los procesos de aprendizaje y brindar aportes capaces de ampliar la perspectiva en la formación docente. En este marco, se indagaron las habilidades socioemocionales de estudiantes de profesorado a partir de la implementación de un cuestionario de autoinforme. Los resultados se estiman promisorios para abrir espacios de reflexión acerca del papel que debería desempeñar este tipo de habilidades en la nueva agenda didáctica. Así, los hallazgos obtenidos desafían a revisar los diseños curriculares que orientan la formación del cuerpo docente para identificar contribuciones capaces de impactar en el desarrollo de sus habilidades socioemocionales y propiciar la conformación de comunidades educativas.

Palabras clave: Habilidades socioemocionales, Comunidad de aprendizaje, Perspectiva integral, Formación docente.

\section{Abstract:}

This study is aimed at describing and analyzing the role that emotions play on socioeconomic skills within an educational community as executed San Jose Primary School Teachers College in Cordoba, Argentina (Profesorados de Educación Inicial y de Educación Primaria del Instituto Superior de Formación Docente San José). This institute possesses a favorable learning environment that surpasses traditional prescriptive curriculums which include the development of socioemotional skills, a topic that has a positive impact on the composition of an educational community. We may ask ourselves, How are teachers preparing for the future in considering such teaching and learning perspectives within their scope of work? How will they broadly exceed the cognitive skills and place socioemotional skills as an important priority of their skillset? This study prompts us to reflect on the complexity of learning and aims to contribute to broadening the teaching horizons of teachers. Within this framework, the socioemotional skills of teachers in training was surveyed through a questionnaire. The results are considered promising in order to open spaces for reflection on the role that this type of skills should play in the new didactic agenda. Thus, the obtained findings challenge us to revise the curricular designs that guide teacher training to identify contributions capable of impacting their socioemotional skills and promote the development of educational communities. The results are considered to be promising since they open spaces for reflection on the role that these types of skills could play in the new didactic agenda. Thus, the obtained findings 
challenge us to revise curricular designs that guide teacher training to identify contributions that will foster the development of their socio-emotional skills and promote the evolution of educational communities.

KEYwords: Socio-Emotional Skills, Learning Community, Integral Perspective, Teacher Training.

\section{INTRODUCCIÓN}

El trabajo que se presenta constituye un avance de un proyecto de tesis doctoral orientado a describir y analizar el rol de las emociones y de las habilidades socioemocionales en el proceso de surgimiento y desarrollo de comunidades de aprendizaje, en el contexto del nivel superior; específicamente los Profesorados de Educación Inicial y Primaria del Instituto Superior de Formación Docente San José (Córdoba, Argentina).

En esta instancia, se atiende de manera especial la trascendencia que adquieren las habilidades socioemocionales en la conformación de las comunidades educativas y, de forma específica, en los aportes que los avances en esta línea de estudio pueden proporcionar a la formación integral del profesorado.

Se entiende que la escuela constituye un ámbito donde se producen aprendizajes que, sin formar parte de los saberes curricularmente prescriptos, son altamente valorables por las implicancias que adquieren para el desempeño en la comunidad. Esta percepción hace pensar que se trata de un ámbito propicio para el desarrollo de ciertas capacidades las cuales pueden influir en la conformación y/o fortalecimiento de comunidades educativas. Por ejemplo, entre estas habilidades o competencias se puede mencionar el aprender a trabajar interdependientemente, ser una persona proactiva, saber cooperar, tolerar la frustración cuando no se logran las metas propuestas, entre otros aprendizajes.

Es pertinente mencionar que, en el sentido de los postulados anteriores, las comunidades de aprendizaje se erigen como una alternativa a una forma de entender la educación que otorga de manera exclusiva a la educación formal y a las instituciones del sistema educativo, la responsabilidad de la formación de niños, niñas, jóvenes y ciudadanía en general (Coll, 2001). Se propone así una visión amplia de la educación y la tendencia creciente a recuperar el sentido original del concepto, el cual se ha ido restringiendo. La idea clave es que la educación es una responsabilidad de la sociedad en su conjunto, cuestión que implica la exigencia de redefinir las funciones, competencias y responsabilidades de los diferentes escenarios y agentes educativos, como también potenciar el compromiso entre todos ellos en tal sentido.

Para ello se parte de las respuestas ofrecidas por un grupo de estudiantes de Nivel Superior al Cuestionario sobre Habilidades Percibidas (Paoloni y Rinaudo, 2015), cuyo análisis se focaliza particularmente en las habilidades consideradas por el modelo de Bar-On sobre Inteligencias no cognitivas (EQ-i) (Gabel, 2005; Bar-On, 2014). Cabe decir que este modelo está compuesto por cinco elementos: el componente intrapersonal, reúne la habilidad de ser consciente, de comprender y relacionarse con otras personas; el componente interpersonal, implica la habilidad para empatizar, ser responsable y poder establecer y mantener vínculos con las demás personas; el componente de manejo de estrés, involucra la habilidad para controlar los impulsos y tolerar las presiones originadas por situaciones estresantes; el componente de estado de ánimo, supone la habilidad para ser optimista y estar satisfecho/a con uno/a mismo/a y con la vida en general; y, por último, el componente de adaptabilidad o ajuste, implica la capacidad de ser flexible ante situaciones cambiantes y crear soluciones pertinentes a los problemas sociales y personales con los cuales se enfrenta la gente (Bar-On, 2000 citado por Gabel, 2005; Bar-On, 2014).

Partiendo del análisis de los datos, se desarrollan algunas derivaciones e implicancias de los resultados para el proceso de formación docente, surgidas a partir de la percepción de la complejidad de los aprendizajes que tienen lugar en el ámbito escolar, entendiendo que el rol docente es clave para la formación integral de la ciudadanía. En este sentido, se comparte la idea que el profesorado debe ubicarse en un lugar de actores destacados en el desarrollo del ecosistema del conocimiento (Manes y Niro, 2016).

Por otra parte, dado que educar y aprender es un proceso complejo de construcción conjunta entre profesorado y alumnado, se considera que de la misma manera que cualquier estudiante sale cambiado al 
final de un curso académico, las secuelas de ese curso dejan también mella en el cuerpo docente. Quienes enseñan también aprenden de las situaciones educativas (Huertas y Montero, 2001), quienes enseñan, nunca terminan de aprender.

Estas consideraciones animan en el estudio de cuestiones propias de la formación del futuro profesorado, específicamente el rol que adquieren las habilidades socioemocionales en el proceso de conformación de comunidades de aprendizaje, como una contribución que favorezca procesos formativos integrales de quienes han tomado como opción personal la docencia.

Quien pretende educar se convierte de alguna manera en responsable del mundo ante quien aprende y eso no implica aprobarlo sino asumirlo conscientemente, porque solo a partir de lo que es, puede ser enmendado (Savater, 1997).

La idea sería que las habilidades socioemocionales son importantes para el bienestar de una sociedad en general, son un cimiento crucial para la construcción de comunidades proactivas. Es importante en tal sentido, promover su desarrollo desde la escuela, pero, para ello, es fundamental que los docentes primero aprendan a identificarlas, valorarlas debidamente y desarrollarlas en ellos mismos. En otras palabras, es importante que este tipo de competencias se desarrolle en el seno de comunidades educativas capaces de impactar en comunidades más amplias, no necesariamente educativas. Así, este estudio explora cómo percibe un grupo de futuras docentes algunas habilidades socioemocionales consideradas por la literatura especializada como fundamentales para el desempeño profesional docente.

\section{MARCo TEÓRICo}

Acerca de las habilidades socioemocionales. La sociedad requiere una amplia variedad de habilidades. La mayoría de las definiciones coinciden en señalar que las competencias ${ }^{[1]}$ son aprendidas o bien susceptibles de aprendizaje y desarrollo.

Para que una persona demuestre competencia ante una determinada tarea, no solo necesita tener dominio de conocimientos conceptuales (saber), procedimentales (saber hacer) y actitudinales (saber ser y estar), sino que también requiere estar motivada para actuar (querer hacer) y deberá tener ciertas características personales y propiedades de contexto favorables para la actuación (poder hacer) (Repetto y Pérez, 2007). Este postulado permite pensar en los aspectos socioemocionales como un factor de incidencia en el desempeño de las personas en diferentes contextos y tareas.

En tal sentido, parece pertinente describir brevemente las dimensiones de las emociones, como una forma de aproximarse a una comprensión más acabada de cómo es posible atender y desarrollar competencias o habilidades socioemocionales. En primer lugar, la dimensión afectiva de la emoción refiere a la experiencia subjetiva que tiene sentido y razón personal. Por eso, se dice que las emociones son estados afectivos subjetivos los cuales provocan que se experimenten ciertos sentimientos en toda su intensidad y calidad ante situaciones, personas, objetos particulares, ya sea pasados, presentes o futuros, reales o imaginarios (Paoloni, 2014).

En segundo lugar, es oportuno señalar que la dimensión fisiológica de la emoción, incluye la participación de los sistemas autonómico y hormonal (Reeve, 1994, citado por Paoloni, 2014), lo cual de alguna manera permite entender que cuando se está emocionado, el cuerpo entra en un estado de activación particular (arousal emocional) que hace que el corazón lata con fuerza, la respiración se acelere, los músculos se tensen, etc.

En tercer lugar, se encuentra la dimensión funcional de la emoción, esta se vincula con los beneficios que las emociones proporcionan en la adaptación del organismo a las características de su entorno. En este sentido vale decir que las emociones permiten ser más efectivos cuando se trata de interactuar con el entorno, favoreciendo la selección de respuestas más apropiadas para cada situación de acuerdo a las metas o propósitos particulares que se hayan planteado. 
Finalmente, cabe mencionar la dimensión expresiva de las emociones, que refiere al componente conductual y social de esta. Resulta pertinente pensar que las emociones son fenómenos sociales por naturaleza y que de manera constante se emiten mensajes emocionales a los demás y se infieren sentimientos privados de las demás personas a través de sus expresiones públicas, mediante la postura, los gestos, las vocalizaciones, la conducta facial.

Atendiendo de manera especial a esta última dimensión de las emociones, Paoloni destaca algunas funciones sociales de las emociones cuyo estudio puede ser significativo por las implicancias en el contexto educativo. Entre ellas se menciona la facilitación de la comunicación de los estados afectivos; la regulación de la manera en que los otros responden a los demás; la promoción de interacciones sociales y la promoción de la conducta prosocial (Paoloni, 2014).

En esta línea de ideas, el modelo de Bar-On de Inteligencias no cognitivas (EQ-i), se asienta en competencias a través de las cuales se intenta explicar cómo una persona se relaciona con las personas que le rodean y con su medio ambiente. Dicho modelo está compuesto por cinco elementos: 1) el componente intrapersonal, que reúne la habilidad de ser consciente, de comprender y relacionarse con otros; 2) el componente interpersonal, que implica la habilidad para empatizar, ser responsable y poder establecer y mantener vínculos con los y las demás; 3) el componente de manejo de estrés, que involucra la habilidad para controlar los impulsos y tolerar las presiones originadas por situaciones estresantes; 4) el componente de estado de ánimo, que supone la habilidad para ser optimista y estar satisfecho con uno/a mismo/a y con la vida en general; y, por último, 5) el componente de adaptabilidad o ajuste, que implica la capacidad de ser flexible ante situaciones cambiantes y crear soluciones pertinentes a los problemas sociales y personales con que uno se enfrenta (Bar-On, 2000 en Gabel, 2005; Bar-On, 2014).

Acerca de las comunidades educativas. Puede decirse que las comunidades de aprendizaje son un instrumento para promover el diálogo intercultural, la cultura de la paz y el desarrollo sostenible.

Sobre las comunidades de aprendizaje, Coll (2001) reconoce que existe un ámbito de conocimientos y experiencias en proceso de articulación y de construcción coherente; sin embargo señala que es posible detectar una serie de elementos en relación con la dirección en que puede orientarse la revisión en profundidad de la educación escolar y de los sistemas de educación formal, que afectan planos y niveles esenciales tales como las aulas, los centros educativos, el contexto social y comunitario, el empleo de las nuevas tecnologías de la información y la comunicación, entre otros.

Partiendo de estos postulados, se considera pertinente revisar algunos aspectos desarrollados desde las comunidades de aprendizaje que interpelan en el tratamiento de miradas más extensas, de propuestas abarcadoras, de procesos educativos integrales. Entre estas cuestiones cabe considerar una visión amplia de la educación; en este contexto es interesante mencionar que las comunidades de aprendizaje encarnan la tendencia creciente a recuperar el sentido amplio original del concepto de educación, que se ha ido restringiendo y limitando. La idea clave es que la educación es una responsabilidad de la sociedad en su conjunto. Esto comporta la exigencia de redefinir las funciones, competencias y responsabilidades de los diferentes escenarios y agentes educativos, como así también potenciar el compromiso entre todos ellos en tal sentido.

Por otra parte, se propone la construcción de un conocimiento colectivo como contexto, plataforma y apoyo a los procesos individuales de aprendizaje, entendiendo que en las comunidades de aprendizaje las personas con diferente nivel de experiencia, pericia y conocimiento aprenden gracias a la colaboración que establecen entre sí, a un proceso de construcción colectivo y a ayudas mutuas que tienen lugar.

Otro de los postulados de las comunidades de aprendizaje propone la superación de las barreras entre la educación formal, no formal e informal, poniendo el acento en la importancia del desarrollo, la socialización y la formación de las personas.

En este marco de ideas, se propone la adopción del concepto de aprendizaje a lo largo de la vida como principio organizativo de la educación y como objetivo comunitario. En coincidencia con principios desarrollados por 
UNESCO (2010), la educación y el aprendizaje se conciben como procesos que tienen lugar desde el nacimiento hasta la muerte y que dependen de las oportunidades ofrecidas, de las opciones y motivaciones individuales y de las posibilidades que encuentra cada persona en su entorno social y comunitario.

Como último punto se señala el potencial de las tecnologías de la información y la comunicación para configurar nuevos espacios y escenarios educativos y para transformar los existentes. En este aspecto, se considera que las comunidades de aprendizaje pueden incrementar su eficacia cuando utilizan las tecnologías de la información y de la comunicación para ampliar las redes de intercambio potenciando el aprendizaje de sus integrantes (Coll, 2001).

Desde esta concepción, las comunidades de aprendizaje constituyen un grupo de personas que se implican activamente en procesos colaborativos, apoyándose en la experiencia y el conocimiento compartido y distribuido entre ellas y en su contexto (Coll, 2001), donde todos y todas tienen algo para enseñar pero también tienen algo para aprender.

Acerca de la dimensión socioemocional en el enseñar y el aprender en comunidad. Un interesante trabajo de investigación sobre la educación en la provincia de Córdoba (Argentina), concluye que el acceso al nivel primario se encuentra universalizado y que forma parte de conquistas culturales y políticas alcanzadas y consolidadas (Gutiérrez, Beltramino, Castro, González y Tosolini, 2013). Esta situación genera otras demandas y propone un nuevo desafío a lograr: que los niños y las niñas concluyan sus estudios en el tiempo previsto, que se reconozcan como sujetos de derechos, que se comprometan en el respeto por las diferencias (Gutiérrez et al., 2013).

Cuando los niños y las niñas se preocupan por sus compañeros/as, piden que se les trate bien, ensayan modos colaborativos de trabajo, construyen opiniones, ponen de manifiesto logros de la escuela que exceden las competencias observadas en muchas evaluaciones estandarizadas.

La escuela actualmente es uno de los escenarios básicos para la socialización de las personas; ya no solo forma en determinados contenidos, sino también en el ejercicio de ciertos procedimientos y técnicas; es el marco donde se aprende el complejo mundo de las relaciones interpersonales, donde se construye buena parte de las relaciones afectivas, donde se adquieren valores y opiniones. (Huertas y Montero, 2001).

Huertas y Montero (2001) sostienen que las personas se construyen en las experiencias de vida que encuentran en su camino y que en esas experiencias no se encuentran disociados los contenidos por temas, los procedimientos por secuencias, ni se etiquetan los afectos en el momento en el que ocurren. Por eso los autores señalan que la sala de clase es un escenario de vida y como tal, en él se entremezclan las enseñanzas, las opiniones, las actitudes y las emociones que fluyen allí donde hay personas.

Sobre esta última cuestión y tomando ideas de Paoloni (2014), es pertinente observar que las emociones son multifacéticas o multidimensionales pues en cada experiencia emocional interviene una diversidad de elementos subjetivos, fisiológicos, funcionales y sociales, actuando de manera coordinada. Por ello, la autora avanza en el pensamiento acerca de que la emoción es un constructo psicológico que integra o resume en un todo coherente, esos componentes de la experiencia humana. De modo que no es posible reducir la emoción a alguna de sus dimensiones constitutivas. Paoloni (Reeve, 1994 citado por Paoloni, 2014) retoma palabras de Reeve en esta línea de ideas, cuando expresa que "la emoción es el director de orquesta de los músicos subjetivos, fisiológicos, funcionales y expresivos que juntos crean una sinfonía de experiencia” (p.89). En consonancia con esta idea, cabe decir que el medio que circundante y la propia existencia genera en las personas necesidades que luego darán lugar a la aparición de motivos. No todas las situaciones contextuales constituyen en sí la razón, el fundamento de la actividad que se ejecuta. Las necesidades entre personas pueden ser las mismas pero los motivos que llevan a la preparación de una actividad pueden ser diferentes. Por eso cabe decir que las vivencias afectivas tienen una dimensión subjetiva, la cual explica por qué diferentes personas, aun teniendo el mismo reflejo cognoscitivo, no tienen las mismas vivencias afectivas al respecto, ya que esta guarda una significativa relación con sus necesidades y motivos, además de una estrecha vinculación con las experiencias vividas por cada persona (Reyes, 2004). 
Por otra parte, y atendiendo a los ambientes educativos, se puede expresar que estos se encuentran impregnados de intensas experiencias emocionales que afectan, tanto de manera positiva como negativa, el aprendizaje y el desempeño, promoviendo o alterando el crecimiento personal de estudiantes y docentes (Paoloni, 2014).

En tal sentido, se estiman relevantes las apreciaciones de Garello y Rinaudo (2012) cuando sostienen que la tarea de guiar al estudiantado de nivel superior desde el nivel inferior de desarrollo académico hasta un nivel superior de habilidad,

conlleva la creación de contextos positivos que promuevan la construcción significativa de conocimientos, el despliegue del aprendizaje autorregulado, el incremento de la motivación personal y el desarrollo de procesos de cognición distribuida, colaborativa y creativa en los futuros expertos de dominios académicos particulares (p.160).

Frente a este marco, resulta necesario revisar ciertas prácticas vinculadas a la formación docente en un sentido que promuevan la producción de saber pedagógico didáctico que ayude a asumir los cambios y a favorecer experiencias de aprendizaje significativas, que cubran competencias cognoscitivas, sociales y emocionales, permitiendo el desarrollo integral de los estudiantes (Gutiérrez et al., 2013; Reimers, 2009).

\section{Procedimientos metodológicos}

\section{Contexto de implementación.}

Se presentan los principales resultados de un estudio con estudiantes del Instituto Superior de Formación Docente ubicado al sur de la provincia de Córdoba, Argentina.

Se trata de una institución educativa de gestión privada, donde se desarrollan los cuatro niveles de enseñanza: Inicial, Primario, Secundario y Superior.

Por otra parte, la institución atiende la formación profesional de estudiantes de la localidad y de la zona de influencia, pues en el ámbito específico del Nivel Superior, donde se cursan estudios correspondientes al Profesorado de Educación Primaria y al Profesorado de Educación Inicial, se manifiesta una importante concurrencia de jóvenes de la localidad como así también de localidades cercanas, conformando una matrícula que actualmente asciende a 93 estudiantes.

\section{Participantes.}

En el estudio participaron 53 estudiantes: 30 alumnas de primer año, 18 alumnas de tercer año y 5 alumnas de cuarto año de los Profesorados de Educación Primaria y de Educación Inicial. La población estuvo constituida por estudiantes mujeres -pues el estudiantado está conformado de este modo-, en su totalidad cuya edad promedio es de 24 años.

Se trata de una muestra intencional, seleccionada teniendo en cuenta las posibilidades de acceso al campo. Los datos fueron recabados entre los meses de febrero y julio de 2015, durante el horario vespertino.

\section{Instrumento.}

Para recolectar la información, se utiliza el Cuestionario sobre Habilidades Percibidas (Paoloni y Rinaudo, 2015). Se trata de un instrumento de autoinforme compuesto por 33 ítems que listan diferentes habilidades percibidas por los y las estudiantes, excepto el ítem 33 que brinda la posibilidad de agregar habilidades no consideradas por el instrumento. 
Para cada habilidad o ítem, cada estudiante debe decidir acerca de tres aspectos: 1) el grado en que percibe haber desarrollado la habilidad mencionada marcando con una cruz o tilde la opción correspondiente (mucha, poca, nada, no sé); 2) si los/as otros/as (pares, docentes, padres, compañeros y compañeras, etc.) consideran que ha desarrollado dicha habilidad (en este caso marca sí, no o no sé, según corresponda); 3) si la habilidad en cuestión es importante para su desempeño en el nivel superior (para lo cual debe seleccionar entre cinco a diez habilidades de la lista y marcarlas circulando el número que las identifica).

Importante precisar que para este artículo en particular se seleccionaron únicamente los resultados relativos al grupo de habilidades socioemocionales, que en el instrumento empleado fueron consideradas con base en el modelo teórico propuesto por Bar-On (2000, por Gabel, 2005).

Es oportuno mencionar que el Cuestionario sobre Habilidades Percibidas constituye un material en etapa de revisión, elaborado en el marco de proyectos institucionales aprobados por diversos organismos de Ciencia y Tecnología de la República Argentina. Se destaca también que no se efectuaron ajustes al cuestionario para que responda a la población con la cual se trabajó sino que este fue aplicado en su versión original.

\section{ANÁLISIS Y DISCUSIÓN DE RESULTADOS}

Para el análisis y discusión de la información recolectada se parte de algunos aspectos que indaga el Cuestionario sobre Habilidades Percibidas; específicamente lo relativo a las socioemocionales según el modelo teórico de referencia. La Tabla 1 presenta los ítems que conforman el grupo de habilidades socioemocionales que valora el cuestionario y los puntajes promedio obtenidos por el grupo de estudiantes en cada uno de ellos. 
TABLA 1

\begin{tabular}{|c|c|c|c|c|c|c|c|c|c|}
\hline \multirow{2}{*}{$N^{\circ}$ item } & \multirow{2}{*}{ Habilidades Socioemocionales } & \multicolumn{2}{|c|}{ Mucha } & \multicolumn{2}{|c|}{ Poca } & \multicolumn{2}{|c|}{ Nada } & \multicolumn{2}{|c|}{ No sé } \\
\hline & & $\mathrm{f}$ & $\%$ & $\mathrm{f}$ & $\%$ & $\mathrm{f}$ & $\%$ & $\mathrm{f}$ & $\%$ \\
\hline 9 & $\begin{array}{l}\text { Percibir y entender las 'señales' } \\
\text { que los demás me comunican } \\
\text { acerca de lo que necesitan, } \\
\text { sienten o desean. }\end{array}$ & 33 & 62,3 & 17 & 32,1 & 2 & 3,8 & 1 & 1,9 \\
\hline 10 & $\begin{array}{l}\text { Realizar tareas al servicio de los } \\
\text { demás (ser servicial). }\end{array}$ & 37 & 69,8 & 13 & 24,5 & 0 & 0 & 3 & 5,7 \\
\hline 11 & $\begin{array}{l}\text { Establecer y mantener relaciones } \\
\text { satisfactorias con los demás } \\
\text { (crear vínculos). }\end{array}$ & 34 & 64,2 & 15 & 28,3 & 0 & 0 & 4 & 7,5 \\
\hline 12 & $\begin{array}{l}\text { Tomar conciencia de mis } \\
\text { sentimientos en el momento en } \\
\text { que los experimento. }\end{array}$ & 23 & 43,4 & 20 & 37,7 & 5 & 9,4 & 5 & 9,4 \\
\hline 13 & $\begin{array}{l}\text { Perseverar ante las dificultades, } \\
\text { tolerar la frustración. }\end{array}$ & 25 & 47,2 & 20 & 37,7 & 7 & 13,2 & 1 & 1,9 \\
\hline 14 & $\begin{array}{l}\text { Percibir los aspectos positivos de } \\
\text { las circunstancias (ser } \\
\text { optimista). }\end{array}$ & 36 & 67,9 & 13 & 24,5 & 3 & 5,7 & 1 & 1,9 \\
\hline 16 & $\begin{array}{l}\text { Acatar normas, acatar órdenes, } \\
\text { aceptar directrices. }\end{array}$ & 37 & 69,8 & 9 & 17 & 2 & 3,8 & 5 & 9,4 \\
\hline 17 & $\begin{array}{l}\text { Expresar mis sentimientos, } \\
\text { manifestar mis emociones. }\end{array}$ & 35 & 66 & 10 & 18,9 & 5 & 9,4 & 3 & 5,7 \\
\hline 18 & $\begin{array}{l}\text { Convencer a los demás, } \\
\text { persuadir a los demás. }\end{array}$ & 8 & 15,1 & 35 & 66 & 4 & 7,5 & 6 & 11,3 \\
\hline 19 & $\begin{array}{l}\text { Liderar, planificar y dirigir } \\
\text { grupos de trabajo. }\end{array}$ & 9 & 17 & 30 & 56,6 & 10 & 18,9 & 4 & 7,5 \\
\hline 21 & Estudiar en grupo. & 17 & 32,1 & 25 & 47,2 & 8 & 15,1 & 3 & 5,7 \\
\hline 23 & $\begin{array}{l}\text { Trabajar en grupo, cooperar, ser } \\
\text { responsable en un equipo. }\end{array}$ & 51 & 96,2 & 2 & 3,8 & 0 & 0 & 0 & 0 \\
\hline 24 & $\begin{array}{l}\text { Ajustar mis sentimientos, } \\
\text { pensamientos y actuaciones a } \\
\text { las condiciones de las } \\
\text { situaciones en las que me } \\
\text { desenvuelvo. }\end{array}$ & 32 & 60,4 & 17 & 32,1 & 0 & 0 & 4 & 7,5 \\
\hline 26 & $\begin{array}{l}\text { Motivarme a mi mismo a } \\
\text { avanzar hacia mis metas. }\end{array}$ & 43 & 81,1 & 9 & 17 & 1 & 1,9 & 0 & 0 \\
\hline 27 & $\begin{array}{l}\text { Planificar mi actuación para el } \\
\text { logro de una meta. }\end{array}$ & 31 & 58,5 & 14 & 26,4 & 2 & 3,8 & 6 & 11,3 \\
\hline 28 & $\begin{array}{l}\text { Tolerar la presión originada por } \\
\text { situaciones estresantes. }\end{array}$ & 19 & 35,8 & 26 & 49,1 & 7 & 13,2 & 1 & 1,9 \\
\hline 29 & $\begin{array}{l}\text { Dominar mis impulsos para } \\
\text { lograr mis objetivos. }\end{array}$ & 26 & 49,1 & 21 & 39,6 & 1 & 1,9 & 5 & 9,4 \\
\hline 30 & $\begin{array}{l}\text { Aceptarme como soy, valorarme } \\
\text { tal cual soy. }\end{array}$ & 45 & 84,9 & 6 & 11,3 & 1 & 1,9 & 1 & 1,9 \\
\hline 31 & $\begin{array}{l}\text { Defender mis derechos, defender } \\
\text { mi punto de vista. }\end{array}$ & 45 & 84,9 & 7 & 13,2 & 0 & 0 & 1 & 1,9 \\
\hline 32 & $\begin{array}{l}\text { Disfrutar de la vida, } \\
\text { experimentar satisfacción en mi } \\
\text { vida. }\end{array}$ & 49 & 92,5 & 4 & 7,5 & 0 & 0 & 0 & 0 \\
\hline
\end{tabular}

Fuente: Paoloni y Rinaudo, 2015

Nota: Frecuencias y puntajes promedio obtenidos para ítems sobre habilidades socioemocionales en el Cuestionario sobre Habilidades Percibidas.

La Tabla 1 reúne los resultados correspondientes a frecuencias y puntajes promedio obtenidos para cada uno de los ítems que valoran la percepción de habilidades socioemocionales en el Cuestionario sobre Habilidades Percibidas (Paoloni y Rinaudo, 2015). Se puede destacar que estos resultados refieren a las respuestas de 53 alumnas de Profesorados de Educación Primaria y Educación Inicial durante el año 2015. 
A partir del modelo propuesto por Bar-On (2014; 2000, en Gabel, 2005), se considera en primer lugar el componente intrapersonal de la inteligencia no cognitiva que reúne la habilidad para conocer y comprender las emociones propias, así como expresar los sentimientos con eficacia (ítems 12 y 17), presentados en la Tabla 1. Al respecto, los resultados sugieren que casi la mitad de las participantes de este estudio $(47,1 \%)$ percibe tener poco o nada desarrollada la habilidad para tomar conciencia de los sentimientos que experimentan en un momento dado. Al respecto, Gardner (1988, citado por Goleman, 2007) expone que la inteligencia intrapersonal es una capacidad vuelta hacia el interior, la cual permite formar un modelo preciso y realista de uno mismo y ser capaz de usar ese modelo para operar eficazmente en la propia vida.

En relación con este aspecto, es pertinente notar que la conciencia de uno mismo, que implica dirigir la atención al mundo interior de pensamientos y sentimientos, constituye un primer paso para la buena conducción. Contar con un enfoque personal, íntimo, hace posible comprender y manejar el mundo interno aun cuando se encuentra conmocionado por elementos perturbadores.

En segundo lugar, respecto de los resultados relativos al componente interpersonal, integrado por habilidades para empatizar, asumir responsabilidad social, establecer y mantener vínculos con los demás (ítems $9,10,11,18,19,22$ y 23), parece alentador el porcentaje del 62\% de alumnas que reconoce tener muy desarrollada la habilidad para empatizar con las demás personas, así como un $64 \%$ que expresa tener mucha habilidad para establecer y mantener vínculos con las y los demás. No obstante, es importante retomar que casi $38 \%$, perciben tener un escaso o nulo desarrollo de la capacidad para empatizar o bien, no saben qué responder al respecto.

Sobre esta cuestión, es importante referirse a lo que afirma Gardner (1988, citado por Goleman, 2007) cuando dice que la inteligencia interpersonal incluye las "capacidades para discernir y responder adecuadamente al humor, el temperamento, las motivaciones y los deseos de los demás” (p. 60). Siguiendo al mismo autor, puede sostenerse que la inteligencia interpersonal constituye la capacidad para comprender a las demás personas, saber qué las motiva, cómo operan, cómo trabajar cooperativamente con ellas y ellos.

En este sentido es interesante retomar postulados de Manes y Niro (2016) quienes, refiriéndose al funcionamiento del cerebro, explican que muchas funciones de dicho órgano están involucradas en permitir tener interacciones sanas. Entre estas funciones se encuentra la empatía, la cual posibilita entender y responder a las experiencias emocionales de las personas. Sobre esta cuestión, los autores agregan que se está predeterminado/a a empatizar y cuando se desarrollan amistades y se cuenta con gente de confianza, los recursos se expanden. Por otra parte, es interesante percatarse de que los lazos positivos y duraderos con otras personas resultan imprescindibles para el bienestar general de los seres humanos, al punto que los vínculos sociales que proporcionan un sentido de pertenencia, protegen contra los sentimientos de soledad, depresión y ansiedad (Manes y Niro, 2016).

En relación con los componentes intra e interpersonal mencionados, es interesante advertir que el aprendizaje social y emocional complementa la parte académica de modo que todo junto conforma la educación integral. Es pertinente añadir algunos aportes de Goleman y Senge (2016) en los cuales desarrollan un análisis de la empatía, donde explican que no basta saber cómo piensan las demás personas, sino que también es preciso preocuparse por ellas y estar dispuestos a ayudarles.

Siendo coherentes con el modelo propuesto por Bar-On, se puede pensar que si alguien tiene dificultades para conectarse con sus emociones y discernir así acerca de las causas que las provocan, cabe preguntarse qué posibilidades tiene de hacer una lectura sensible de las emociones, sentimientos, necesidades o estados anímicos del resto de personas. Las respuestas a las que se hace referencia pertenecen a futuros docentes...

Por otra parte, y en esta misma línea de ideas, se considera aquí una de las cuatro capacidades que para Goleman (2007) constituyen los componentes de la inteligencia interpersonal. Se trata de la organización de grupos, esencial en un líder; esta habilidad incluye esfuerzos iniciadores y coordinadores de una red de personas, persuadiendo a sus integrantes en el trabajo hacia un objetivo común. 
Los resultados obtenidos -de acuerdo con los ítems 18 y 19- arrojan que los porcentajes de alumnas que admiten haber desarrollado poco y nada estas habilidades son entre un $75 \%$ y $80 \%$ del total; aspecto que llama la atención por la importancia de estas para la tarea de enseñar especialmente en los nuevos escenarios con que futuros profesionales docentes se enfrentarán.

En el momento de conformar un equipo de estudio es fundamental plantearse seriamente su necesidad, distinguir su estructura, los roles y tareas por asignar conforme al proyecto que se lleve adelante, dado que la buena organización de un grupo desde su origen, es fundamental para su éxito. Se puede recalcar entonces que el trabajo en equipo supone el despliegue de múltiples destrezas sociales tales como empatía, cooperación, comunicación, solidaridad y liderazgo.

En este sentido, el liderazgo es tanto influenciar a personas para contribuir al trabajo del grupo como coordinar actividades necesarias para lograr los objetivos propuestos. En el proceso de acercarse a las metas deseadas, su condición no es el ejercicio de la autoridad absoluta sino la tarea de conocer y comprender los valores y las opiniones de las personas con las cuales se trabaja. De aquí que la persona que ejerce el liderazgo deba posicionarse como una más del grupo, pertenecer al conjunto más que estar por encima, constituirse más en la regla que en la excepción (Manes y Niro, 2016). Se entiende que el rol de la persona líder implica una tarea compleja pero sumamente necesaria para poder avanzar en la consecución de objetivos comunes; por otra parte, se advierte que en muchos contextos esta tarea se desvirtúa y se percibe como un intento de imposición personal. Los aportes teóricos citados, dan cuenta de una perspectiva diferente que sería interesante dar a conocer al estudiantado de las carreras del profesorado de Educación Inicial y de Educación Primaria.

En tercer término y considerando particularmente el componente de manejo de estrés, que involucra la habilidad para tolerar las presiones y controlar los impulsos (ítems 13, 16, 28 y 29), los resultados muestran que un $49 \%$ de las participantes admite no tener dificultades para dominar sus impulsos para el logro de las metas, mientras que entre un $50 \%$ y un $60 \%$ considera tener poco o nada desarrolladas las habilidades para perseverar ante las dificultades y tolerar las presiones originadas por situaciones estresantes.

Sobre esta cuestión, importante retomar lo dicho anteriormente al respecto por Goleman (2007) cuando afirma que "es la combinación de talento razonable y la capacidad de seguir adelante ante las derrotas lo que conduce al éxito" (p. 115). En este sentido, es interesante atender a la idea de autoeficacia, es decir la creencia de que uno tiene dominio sobre los acontecimientos de su vida y puede aceptar los desafíos tal como se le presentan. Desarrollar una competencia de cualquier clase refuerza la noción de autoeficacia y hace que la persona esté más dispuesta a correr riesgos y a buscar mayores desafíos. En esta misma línea de pensamiento, Bandura (1988 citado por Goleman, 2007), expresa que las convicciones de la gente respecto de sus habilidades tienen un profundo efecto en esas habilidades y que las personas con una idea de autoeficacia se recuperan de los fracasos y abordan las tareas en función de cómo manejarlas en lugar de preocuparse por lo que pueda salir mal.

A partir de lo anterior resulta importante ayudar a desarrollar ideas de autoeficacia en estudiantes del Profesorado de Educación Inicial y del Profesorado de Educación Primaria como una importante contribución a sus logros en el ámbito académico, pero también en sus desempeños en otros contextos en los cuales se vean implicados. Vale la pena pensar también que tales aprendizajes pueden tener impacto en las aulas y en el estudiantado con el que trabajen en el futuro, favoreciendo el desarrollo de una mirada centrada en las posibilidades de logro de cada uno y de revertir los resultados adversos que puedan ocurrir.

En cuarto lugar, se atiende al componente de estado de ánimo -optimismo y satisfacción- (ítems 26, 30, 31 y 32). Las habilidades que integran esta categoría obtuvieron porcentajes elevados, esto supone que más del $80 \%$ de las estudiantes que participaron de este estudio, consideran que han desarrollado de modo importante habilidades que les permiten generar estados de ánimo propicios para avanzar hacia sus metas, estar satisfechas con ellas mismas y con la vida y percibir los aspectos positivos de las circunstancias del entorno. Ser optimista implica una gran fortaleza en tanto significa tener grandes expectativas de que las cosas saldrán bien a pesar de 
los contratiempos y las frustraciones. Desde el punto de vista de la inteligencia emocional, el optimismo es una actitud que evita que la gente caiga en la apatía, la desesperanza o la depresión ante la adversidad (Goleman, 2007). El buen humor favorece así la capacidad de pensar con flexibilidad y con mayor complejidad haciendo que resulte más fácil encontrar soluciones a los problemas, sean estos de carácter interpersonal o intelectual.

Estas respuestas de las estudiantes constituyen un dato interesante que podría incidir favorablemente en el trabajo sobre aspectos analizados anteriormente y que aparecen como tareas pendientes, como aprendizajes que deberían desplegar para un desarrollo personal y profesional óptimo.

Finalmente, en quinto lugar, se considera el componente de adaptación-ajuste, que refiere a la capacidad de la persona para evaluar correctamente la realidad y ajustarse de manera eficiente a nuevas situaciones, así como a la habilidad para crear soluciones adecuadas a los problemas diarios (ítems 24 y 27). En el marco del estudio, los resultados sugieren que un $60 \%$ de las participantes, percibe que ha desarrollado convenientemente habilidades para planificar su actuación para el logro de metas y adaptarse a las situaciones cambiantes del entorno; sin duda, habilidades importantes para enfrentar los retos de una sociedad que requiere cada vez más este componente de flexibilidad y determinación en el comportamiento de las personas que la integran.

Respecto de estos resultados, se puede decir que, de alguna manera, para los seres humanos la supervivencia depende en gran medida de un funcionamiento social efectivo. En este sentido, la propensión a la agresividad impulsiva parece estar asociada con una falta de autocontrol sobre ciertas respuestas emocionales negativas y una incapacidad para comprender las consecuencias negativas de este comportamiento (Manes y Niro, 2015). No se trata de desactivar las perturbaciones de las emociones, del contexto para evitar caer en errores, sino de tomar conciencia de cómo esos atajos están operando y saber identificar en qué situaciones no debe tenérselos en cuenta.

Autores como Huertas y Montero (2001), atienden a este componente de adaptabilidad-ajuste en relación con los contextos educativos y sugieren que para promover las habilidades que están en su base, es preciso crear situaciones de enseñanza en donde se fomenten oportunidades para que las personas compartan y construyan un mismo marco de referencia el cual les haga enriquecer sus conocimientos anteriores. Lo necesario entonces para el aprendizaje, es el conocimiento mutuo y la intersubjetividad, el intercambio de pareceres, la confluencia.

Estas ideas invitan a pensar las oportunidades de aprendizaje sucedidas a partir de actividades con otras personas, que implican entre otros aspectos, el desarrollo de habilidades vinculadas con la capacidad de adaptarse y de generar comportamientos favorables para la tarea grupal.

\section{Conclusiones}

Si bien los resultados presentados en este artículo son preliminares, las premisas que sugieren no dejan de ser por demás interesantes. Los hallazgos obtenidos invitan a reflexionar sobre las oportunidades de consolidar en la escuela un modelo que atienda el proceso educativo del estudiantado, ayudando al grupo docente a prepararse de manera más adecuada para aquello que van a enseñar.

Una mirada retrospectiva sobre la educación permite advertir que se ha colocado en un lugar de privilegio la individualidad del alumnado y el contenido, escasamente relacionado con el contexto de aprendizaje, con las personas que acompañan esa instancia, con la subjetividad de quien aprende (Coll, 2009; Perkins, 2010; Pogré y Lombardi, 2004; Robinson, 2013). Sin embargo, con el devenir de estudios al respecto, se han llegado a proponer concepciones más integrales de la educación y del proceso de enseñanza y aprendizaje específicamente. En este sentido, se plantea que el aprendizaje es un proceso que ocurre desde la cuna hasta la tumba (UNESCO, 2010), en el que no es posible o deseable prescindir del contexto cultural, económico, social e histórico de la persona que aprende (Cole, 1999; Gardner, 2013; Perkins, 2010; Pineau, 2007; Pogré y Lombardi, 2004). En otras palabras: 
(...) el paradigma de enseñanza-aprendizaje centrado en los contenidos ha sido sustituido por otro centrado en el estudiante, que propone el desarrollo de competencias para la formación de ciudadanos autónomos, capaces de emitir juicios y tomar decisiones responsables, con base en el conocimiento y razonamiento, que les permita aprender de manera continua y resolver problemas más allá del contexto escolar, y en diferentes etapas de su vida (Gaeta, 2014, p. 33).

En esta línea de pensamiento, existe acuerdo en sostener que para alcanzar un nivel de desarrollo pertinente de sus posibilidades, las personas necesitarán niveles altísimos de confianza en sí mismas, tendrán que ser flexibles, capaces de utilizar su creatividad natural, y conscientes de sus propias fortalezas y debilidades. También deberán tener cada vez mayor conciencia de sí mismos a nivel emocional e intelectual, además de ser capaces de establecer relaciones de manera rápida, efectiva y a menudo virtual con los demás. Por eso puede decirse que la educación encuentra así nuevos espacios de conocimiento a los cuales atender (Gerver, 2010).

Esta forma de concebir el aprendizaje condice con una forma de entenderlo más allá de muros de las instituciones educativas y de los trayectos educativos durante la formación de grado. Parece oportuno atender a que se vive en la sociedad del aprendizaje continuo (Pozo y Monereo, 1999), de manera que la educación obligatoria y posobligatoria cada vez se prolongan más, por la movilidad profesional y la aparición de nuevos perfiles laborales, por lo que parece acertado decir que será necesario educarse a lo largo de toda la vida. Si, como se ha destacado, los procesos educativos tienen un carácter continuo y permanente y no se agotan en el marco escolar, se debe reconocer que los aprendizajes de las personas, no se reducen a los ofrecidos en la educación formal (Flecha y Tortajada, 1999; Tedesco, 2009), sino que estas deberán contar con habilidades y estrategias que posibiliten nuevas experiencias de aprendizaje.

Se asume que no se trata de cuestiones simples sino de un sofisticado proceso de aprendizaje mutuo, que implica cambios en el modo de percibir y de comportarse, posibilitado por un sentido de asociación de responsabilidades ante una situación compleja (Goleman y Senge, 2016).

Se entiende que la escuela no puede establecer por sí sola una jerarquía común de saberes, pasiones y valores, pero sí puede hacer dialogar los diversos mundos que habitan los niños, niñas y adolescentes. La escuela necesita volver a pensar qué sienten sus alumnos, qué pasiones los recorren, con qué palabras nombran sus mundos (Gagliano, 2007).

El profesorado es la piedra angular para iniciar o consolidar este proceso de cambio hacia una perspectiva educativa integral. Si se quiere educar desde una visión integradora que considere a las habilidades socioemocionales como importantes baluartes en los aprendizajes académicos construidos por las y los estudiantes, se debe comenzar por las y los profesores, y qué mejor que hacerlo mientras se están formando para ser docentes. Desde esta perspectiva, han de estar integrados en comunidades donde se ayuden unos a otros. De modo que debe estimularse la creación de redes cooperativas verdaderamente sólidas que transformen la cultura de la escuela mediante la colaboración permanente y la constante innovación (Goleman y Senge, 2016).

Sobre esta materia, Goleman (2003) invita a pensar en lo interesante que sería que cada escuela contara con un docente cuya conducta ejemplificara el amor y la compasión. Profundiza su idea al explicar que en las escuelas existen muchos docentes de este tipo que, lamentablemente, no son considerados como modelos. Entonces, lo que se necesita es alentar estas actitudes en el profesorado que no las posea y el apoyo institucional necesario para llevar a cabo este proceso.

Se comprende que es posible ofrecer más espacios y oportunidades en los entornos educativos para la promoción y la construcción de nuevas perspectivas que reconozcan las posibilidades de quienes habitan las aulas y las escuelas. En los contextos educativos se observan múltiples limitaciones, topes y prohibiciones en el momento de enseñar y de aprender, lo que pone en evidencia la necesidad de construir contextos educativos que atiendan la particularidad y la complejidad de los procesos cognitivos y sociales que tienen lugar (Elisondo, 2015).

Las respuestas proporcionadas por las estudiantes permiten la reflexión acerca de que la educación, como sostenía Paulo Freire, es ante todo un problema político que exige pensar qué tipo de sociedad se quiere. En 
esta línea de ideas, se puede afirmar que la educación puede ayudar a fortalecer la ciudadanía, la democracia, la convivencia cuando se dan ciertas condiciones tales como una educación como práctica de la libertad, volcada al bien común y a la promoción de las personas (Ander-Egg, 2008).

Se concibe que las escuelas deben facilitar que el estudiantado desarrolle competencias ciudadanas congruentes con formas pacíficas de asociación y de resolución de conflictos, de legalidad e institucionalidad democráticas y con vivir en el respeto a los derechos humanos de todas las personas (Reimers, 2009).

Las ideas desarrolladas invitan a pensar así la formación docente como una instancia fundamental en el trayecto de aprendizaje de los educadores, con fuertes implicancias personales y sociales. Se necesita cuidar, sostener y profundizar los logros alcanzados con acciones que atiendan aspectos que, quizás hasta el momento, no se han colocado en el eje del debate con el énfasis que requieren. Se piensa aquí en la importancia de atender al desarrollo de las habilidades socioemocionales de futuros docentes como un factor de notable incidencia en el surgimiento y el desarrollo de comunidades educativas venideras.

\section{REFERENCIAS}

Ander-Egg, E. (2008) La ciudad educadora. Córdoba: Brujas.

Bar-On, R. (2014). Workshop sobre Inteligencia Emocional. Recuperado de https://bit.ly/2W02Ma6

Cole, M. (1999) Psicología cultural. Madrid: Ediciones Morata.

Coll, C. (2001). Las comunidades de aprendizaje y el futuro de la educación: el punto de vista del Fórum Universal de las Culturas. Simposio Internacional sobre Comunidades de Aprendizaje, Barcelona.

Coll, C. (2009) Enseñar y aprender en el siglo XXI: el sentido de los aprendizajes escolares. En A. Marchesi, J.C. Tedesco y C. Coll (Coords.), Calidad, equidad y reformas en la enseñanza (pp. 101-112). Madrid: Fundación Santillana, OEI.

Elisondo, R. (2015). La creatividad como perspectiva educativa. Cinco ideas para pensar los contextos creativos de enseñanza y aprendizaje. Revista Actualidades Investigativas en Educación, 15(3), 1-23. doi: http:// dx.doi.org/10.15517/aie.v15i3.20904

Flecha, R. y Tortajada, I. (1999). Retos y salidas educativas en la entrada de siglo. En F. Imbernón (Coord.), La Educación en el siglo XXI. Los restos del futuro inmediato (pp. 13-180). Barcelona: Graó.

Gabel, R. (2005). Inteligencia emocional: perspectivas y aplicaciones ocupacionales. Lima: Universidad ESAN.

Gaeta, M.L. (2014) Autorregulación del aprendizaje y su promoción en el contexto del aula. En P.V. Paoloni, M.C. Rinaudo y A. González (Comps.) Cuestiones en Psicología Educacional: perspectivas teóricas, metodológicas y estudios de campo (pp.33-57). Tenerife: Sociedad Latinoamericana de Comunicación Social (SLCS).

Gagliano, R. (2007) Puertas y puentes de escuelas situadas. Acerca de la luz que atraviesa el prisma de la forma escolar. En R. Baquero, G. Diker y G. Frigerio (Comps.) Las formas de lo escolar (pp. 245-259). Buenos Aires: Del Estante.

Gardner, H. (2013) Las cinco mentes del futuro. Buenos Aires: Paidós.

Garello, V.y Rinaudo, M. C. (2012). Rasgos del contexto para la promoción del desarrollo académico y la creatividad. Estudio de diseño con estudiantes universitarios. Revista Iberoamericana sobre Calidad, Eficacia y Cambio en Educación, 10(2), 159-179.

Gerver, R. (2010) Crear hoy la escuela del mañana. Ediciones SM.

Goleman, D. (2003) Emociones destructivas. Buenos Aires: Vergara.

Goleman, D. (2007) La inteligencia emocional. Buenos Aires: Vergara

Goleman, D. y Senge, P. (2016) Triple focus. Barcelona: Ediciones B.

Gutiérrez, G. M., Beltramino, L., Castro, E., González, E. y Tosolini, M. (2013) El nivel primario en Córdoba: análisis de sus tendencias y transformaciones: 2003-2013. Córdoba: Unión de Educadores de la Provincia de Córdoba.

Huertas, J.A. y Montero, I. (2001) La interacción en el aula. Buenos Aires: Aique.

Manes, F. y Niro, M. (2015) Usar el cerebro. Buenos Aires: Planeta. 
Manes, F. y Niro, M. (2016) El cerebro argentino. Buenos Aires: Planeta.

Paoloni, P.V. (2014) El papel de las emociones en los aprendizajes académicos. En P.V. Paoloni, M.C. Rinaudo y A. González (Comps.) Cuestiones en Psicología Educacional: perspectivas teóricas, metodológicas y estudios de campo (pp. 83-131). Tenerife: Sociedad Latinoamericana de Comunicación Social (SLCS).

Paoloni, P.V. y Rinaudo, M.C. (2015). Cuestionario sobre Percepción de Habilidades. Argentina: Universidad Nacional de Río Cuarto.

Perkins, D. (2010) El aprendizaje pleno. Principios de la enseñanza para transformar la educación. Buenos Aires: Paidós.

Pineau, P. (2007) Algunas ideas sobre el triunfo pasado, la crisis actual y las posibilidades futuras de la forma escolar. En R. Baquero, G. Diker y G. Frigerio (Comps.) Las formas de lo escolar (pp. 33-44). Buenos Aires: Del Estante.

Pogré, P. y Lombardi, G. (2004) Escuelas que enseñan a pensar: enseñanza para la comprensión, un marco teórico para la acción. Buenos Aires: Papers Editores.

Pozo, J.I. y Monereo, C. (1999). El aprendizaje estratégico. Barcelona: Santillana.

Reimers, F. (2009) Educar para la paz y la ciudadanía en América Latina. En A. Marchesi, J.C. Tedesco y C. Coll (Coord.). Calidad, equidad y reformas en la enseñanza (pp. 125-141). Madrid: Fundación Santillana, OEI.

Repetto Talavera, E. y Pérez, J.C. (2007) Formación en competencias socioemocionales a través de las prácticas en empresas. Revista Europea de Formación Profesional, 40, 92-112

Reyes, D. J. (2004) La esfera emocional y su relación con el proceso de aprendizaje. Recuperado de https:// bit.ly/2Z5GK7Z

Robinson, K. (2013) El elemento. Buenos Aires: Conecta.

Savater, F. (1997). El valor de educar. Barcelona: Ariel.

Tedesco, J. C. (2009) Educar en la sociedad del conocimiento. Buenos Aires: Fondo de Cultura Económica.

UNESCO (2010) Informe Mundial sobre el aprendizaje y la educación de adultos. Organización de las Naciones Unidas para la Educación, la Ciencia y la Cultura. Hamburgo: UIL.

\section{Notas}

[1] Habilidades y competencias son términos relacionados, que refieren a disposiciones del ser humano, que se vinculan con una actividad o campo de actividades. Para algunos autores una competencia es más general que una habilidad. Otros, sin embargo, las consideran indistintamente. En este trabajo se emplean los conceptos de manera indistinta.

\section{BY-NC-ND}

